\section{La gamificación como recurso telemático en la comunicación empresarial en tiempos de pandemia}

\section{Gamification as a telematic resource in business communication in times of pandemic}

https://doi.org/10.18566/comunica.n44.a04

Recibido: 17 de diciembre de 2020

Aceptado: 25 de marzo de 2021

\section{Resumen}

Este trabajo tiene como objetivo conocer, desde el punto de vista de los directivos de las empresas pequeñas y medianas con amplia utilización de las tecnologías digitales, si el alejamiento físico de las personas como consecuencia del teletrabajo (en rápido crecimiento por efectos de la pandemia de COVID-19) dificulta las relaciones interpersonales y el conocimiento de los objetivos y valores estratégicos de la empresa, y si ese mismo equipo directivo conoce y en qué grado utiliza la gamificación como posible herramienta para mejorar la comunicación interna con el fin de paliar o solucionar dicha situación.

Para este estudio exploratorio se ha adoptado una perspectiva metodológica cualitativa, basada en una revisión de la bibliografía sobre el papel de la gamificación en la comunicación interna de las empresas y utilizando entrevistas en profundidad, semiestructuradas con preguntas abiertas y cerradas a los directivos de un grupo de pequeñas y medianas empresas que están en el ámbito de este análisis.

Los resultados muestran que todavía no existe clara conciencia ni cuantificación de las consecuencias que produce el alejamiento físico producido por el teletrabajo ni se han adoptado medidas basadas en la gamificación para disminuir su potencial efecto. En la medida que se vaya consolidando el teletrabajo y se puedan medir estos efectos en las comunicación

número 44

Enero - junio

2021 | pp. 57-75

\section{Javier Fábregas Grau}

Doctorando en

Comunicación Digital y

Nuevas Tecnologías de la

Universidad Abad Oliba CEU.

jav.fabregas.ce@ceindo.

ceu.es

\section{Santiago Tejedor}

Doctor en Periodismo y

Ciencias de la Comunicación

de la Universidad Autónoma

de Barcelona (UAB).

Coordinador del Gabinete

de Comunicación y

Educación de la UAB.

santiago.tejedor@uab.cat

https: / / orcid.org/0000-

0002-5539-9800

\section{Javier Salla García}

Doctor en Ciencias Jurídicas de la Universidad Autónoma de Barcelona e investigador del Observatorio

Iberoamericano de la Comunicación de la Facultad de Ciencias de la Comunicación de la UAB (proyecto competitivo). xavier.salla@uab.cat http: / / orcid.org/0000-00032946-969X 
relaciones interpersonales y el sentido de pertenencia a la empresa, la gamificación podrá cumplir las expectativas que está despertando y aportar, junto a técnicas de liderazgo, gestión del talento y principios de la RSC (Responsabilidad Social Corporativa), mayor cohesión y valor a las pymes.

\section{Abstract}

The aim of this paper is to find out, from the point of view of managers of small and medium-sized enterprises that make extensive use of digital technologies, whether the physical remoteness of people as a result of teleworking (which is growing rapidly due to the effects of the COVID-19 pandemic) hinders interpersonal relations and knowledge of the company's strategic objectives and values, and whether this same management team is aware of and to what extent it uses gamification as a possible tool for improving internal communication to alleviate or solve this situation.

For this exploratory study, a qualitative methodological perspective was adopted, based on a review of the literature on the role of gamification in the internal communication of companies and using in-depth, semistructured interviews with open and closed questions to the managers of a group of small and medium-sized companies within the scope of the study.

The results show that there is still no clear awareness or quantification of the consequences of physical remoteness caused by teleworking, nor have gamification-based measures been adopted to reduce its potential effect. As teleworking becomes more established and these effects on interpersonal relations and the sense of belonging to the company can be measured, gamification will be able to meet the expectations it is arousing and, together with leadership techniques, talent management and CSR (Corporate Social Responsibility) principles, bring greater cohesion and value to SMES.

\section{Introducción}

Cuanto más avanza la tecnología y más se introduce esta en la cotidianeidad de los ciudadanos, más importancia supone la utilización de la comunicación rápida e inmediata, capaz de llevar a muchos el mismo mensaje o la misma información. Este aspecto cabe aplicarlo también al ámbito de la empresa, donde la comunicación deviene fundamental para conocer la situación de aquella en todo momento, cuáles son sus objetivos estratégicos a corto y largo plazo y, en especial, para facilitar la cohesión de sus empleados, de forma que se pueda infundir una cultura que favorezca la homogeneidad de criterios de actuación y decisión en todas las actividades del día a día y se fomente el sentido de pertenencia.

\section{Palabras clave}

Teletrabajo, comunicación interna, cohesión, comunicación virtual, gamificación, pequeña y mediana empresa.

\section{Keywords}

Teleworking, corporate communication, cohesion, virtual communication, gamification, small and medium-sized businesses. Márquez, literary journalism, animal turn. 
Este proceso de difusión de la información e interrelación de las personas se ha visto dificultado con el alejamiento físico de los miembros de las estructuras organizativas cuando se ha puesto en marcha la utilización del teletrabajo, en especial y de manera obligada y muy rápida como consecuencia de la pandemia.

Por su reducida dimensión, a las pequeñas y medianas empresas (pymes) parece que les debería resultar mucho más fácil y ágil disponer de esquemas de comunicación e información fluida entre todos sus componentes en relación con los datos clave que permitan conocer la situación y los valores estratégicos de la compañía, de suerte que un mayor alineamiento de sus miembros incremente la motivación y el compromiso (engagement), y como consecuencia mejore la productividad, la eficiencia y, por ende, la rentabilidad, o en algunos casos ayude a la propia supervivencia de la empresa.

Sin embargo, la realidad no es así y en general las pymes carecen de una metodología o esquema establecido para ello, por lo que en la mayoría de los casos se pierde una herramienta que puede ser no solo muy útil, sino incluso clave para un mejor funcionamiento operativo y para la identificación de las personas con la empresa, su implicación en la estrategia y su mayor satisfacción (e incluso felicidad).

La aparición de la pandemia de COVID-19 a principios del año 2020 ha incrementado rápidamente en las empresas, cuya actividad y funciones lo permiten el trabajo en remoto o teletrabajo como consecuencia de las restricciones de movilidad y distanciamiento social impuestas por la mayoría de los gobiernos del mundo. Ello ha supuesto un alejamiento físico de las personas de su entorno empresarial habitual y de sus compañeros de trabajo, lo que puede provocar más dificultad en la relación entre aquellas y una pérdida de conocimiento sobre la situación de la compañía y de sus objetivos y valores estratégicos, con pérdida del sentido de pertenencia.

Parecía, pues, oportuno acometer este trabajo, consistente en una aproximación experimental preliminar para conocer de primera mano esta posible sensación de pérdida de cohesión entre los miembros de una empresa (tanto entre ellos como de la dirección con sus empleados) y si la gamificación puede resultar una herramienta útil y relevante en estos momentos, cuando la pandemia sigue alimentando los distanciamientos físicos y el trabajo en remoto.

Para este fin se ha realizado un estudio limitado a 13 empresas mediante 32 entrevistas en profundidad a los equipos directivos, cuyos resultados deben motivar futuros trabajos de investigación más amplios y dirigidos a objetivos más concretos. 


\section{Metodología}

Por su finalidad, el presente trabajo pretende detectar precozmente si el teletrabajo está alejando a las personas de sus compañeros y de la empresa, haciendo que disminuya el conocimiento sobre su situación por la pérdida de información y que se vayan diluyendo la implicación con ella y la cohesión social con los demás trabajadores; y, además, qué papel puede jugar la gamificación para mitigar estos efectos.

Se trata de un trabajo limitado de investigación experimental preliminar y para su realización se ha utilizado, básicamente, un método cualitativo mediante la investigación descriptiva y entrevistas en profundidad. Las entrevistas han sido semiestructuradas, con 12 preguntas, tres de ellas abiertas y nueve cerradas. Las preguntas abiertas pretendían fomentar la reflexión de índole cualitativa y verificar la coincidencia de expresiones en una misma empresa o colectivo.

Los resultados de las entrevistas han sido analizados de forma manual, por cuanto la estructura del cuestionario dificultaba el uso de herramientas de investigación cualitativa (por ejemplo, Nvivo) y su reducido número permitía un tratamiento individualizado (en hojas Excel), sin tener que recurrir a programas estadísticos complejos.

La selección de las pymes entrevistadas ha sido aleatoria dentro del sector servicios con utilización intensiva de tecnologías digitales, cuyo objeto social permite con mayor amplitud la utilización del teletrabajo (incluso antes de la pandemia).

\section{Contenido y análisis de resultados}

Antes de mostrar y analizar los resultados obtenidos cabe hacer algunas consideraciones previas.

\section{Sobre la comunicación interna en la estrategia de la empresa}

El estudio se ha hecho con pymes: ${ }^{1}$ menos de 50 trabajadores (pequeñas) o menos de 250 trabajadores (medianas), las cuales suponen el 99,8 \% de las empresas españolas, ${ }^{2}$ para que en una futura investigación de mayor población estadística pueda generalizarse al tipo de empresas más frecuente en España.

Entendiendo el mercado del sector al que pertenecen las empresas analizadas como globalizado hace ya unos años, en un entorno VUCA, ${ }^{3}$ cuantificado según un estudio del Boston Consulting Group de 2013 (Jones y

1 Dos de ellas superan ligeramente los 250 trabajadores.

2 Según clasificación del BOE 10612 de 2013 y Banco de España, en su circular 4/2013 de 27 de septiembre.

3 VUCA es un acrónimo del US Army War College relacionado con estrategia bajo incertidumbre (Courtney et al.) 
Brazzel, 2006), la mitad de los trimestres más turbulentos financieramente en los últimos 30 años se ha producido desde 2002. El escenario VUCA es hoy considerado como el nuevo normal (new normal) (Lawrence, 2013) y está cambiando profundamente no solo las organizaciones empresariales, sino también las habilidades y actitudes que deben tener los nuevos dirigentes y líderes de las compañías. Y para ello deberán poner especial atención en las comunicaciones y dotarse de las herramientas más eficaces para mantener la viabilidad de sus proyectos empresariales (Kingsinger y Walch, 2012).

La mayor parte del tiempo de un líder o directivo (Rosser, (2003), sociólogo y experto en prácticas de ventas y negociación lo estima en el 80 \%) lo destina a transmitir directrices, coordinar procesos entre diferentes áreas, delegar funciones, motivar o integrar a su equipo, resolver conflictos interpersonales o interdepartamentales, crear compromisos de rentabilidad, etc., todas ellas funciones basadas en acciones de lenguaje estableciendo comunicaciones.

La pandemia de COVID-19 ha obligado a los gobiernos de casi todos los países a tomar medidas de confinamiento para la población en sus domicilios particulares; en muchos casos no han podido acceder a los locales de las empresas en las que trabajan, y estas han debido cesar su actividad o bien a desarrollar sus labores, total o parcialmente desde los domicilios de sus colaboradores.

En definitiva, a un escenario ya convulso (VUCA) se le ha añadido un nuevo condicionante (COVID-19), lo que ha obligado a las empresas a un esfuerzo adicional para llevar a cabo su proyecto empresarial. Es decir, que a la necesidad de disponer de mayores habilidades comunicativas, ahora se le suma el hecho de tener que ejecutarlas a distancia.

Si bien no se dispone todavía de datos totalmente elaborados, el Instituto Nacional de Estadística de España (Boletín Informativo, febrero 2020) afirma que, en España en el año 2019, el 4,8 \% de las personas trabajaban normalmente (o al menos la mitad de los días) desde sus casas, cifra muy inferior a países europeos que se sitúan en más del 10 \% (Países Bajos 14 \% y Finlandia 13,3 \%). La empresa de recursos humanos Randstad (febrero 2021), situaba este dato en el 16,2 \% en el segundo trimestre del 2020 (más de 3 millones de personas), es decir casi 3 veces y media más de forma súbita, si bien en el último trimestre del año, este porcentaje ha descendido hasta el 9,9\%.

La digitalización está cambiando drásticamente la manera de utilizar la comunicación; las personas y las empresas acceden a plataformas de intercomunicación y utilizan constantemente la tecnología para intercambiar información o conocimiento. Esta nueva forma de comunicación está suponiendo un impacto en los ámbitos socioculturales y económicos; algunos 
autores opinan que internet, las webs, los social media, las redes sociales, etc., están transformando lo virtual en una "inteligencia colectiva" vinculada a la comunicación digital, que Lévy define como "una inteligencia repartida en todas partes, valorizada constantemente, coordinada en tiempo real, que conduce a una movilización efectiva de las competencias" (2004, p. 20).

Así, las habilidades comunicacionales y las herramientas resultan elementos clave en los procesos de evolución de las empresas en entornos de alta turbulencia y complejidad que ya se tienen como "normales". Por tanto, cualquier avance en la productividad de la dirección pasa por diseñar conversaciones directivas más eficaces o instrumentos que, basados en las nuevas tecnologías, permitan obtener incluso mejores resultados en los procesos de comunicación y transmisión de información.

Para ello, en este trabajo se plantea analizar la existencia de un alejamiento relacional entre las personas, paralelo al alejamiento físico que produce el trabajo en remoto, además del uso de la gamificación como herramienta eficaz a disposición del líder o directivo, tanto para interactuar con el equipo como para que la utilicen entre ellos, con el fin de conseguir un mayor alineamiento de los miembros de la organización con los objetivos (que pueden ser cambiantes en función del momento), extender y homogeneizar la cultura de la empresa (que debe ser adaptable a las circunstancias) y compartir la misma visión (objetivo último de la empresa que debe permanecer de forma constante como referencia de actuación y en la toma de decisiones).

\section{Sobre la gamificación. Concepto}

Se ha elegido el término "gamificación" (adaptación de "gamification" en el ámbito anglosajón como derivado de la palabra "game", traducible por juego) para definir una tendencia que consiste en integrar los elementos y las dinámicas de juego en entornos no lúdicos. No existe un nombre formalmente aceptado para denominar esta actividad, ya que la propia RAE (Real Academia Española) no recoge en su diccionario las palabras gamificación, jueguización, juguetización o ludificación. También se podría optar por la utilización de varias palabras como mecánicas del juego, que quizá, desde el punto de vista comercial resulte excesivamente largo.

A efectos de este trabajo utilizaremos el término (ampliamente adoptado en internet y en los círculos profesionales) gamificación equivalente al término inglés gamification.

Gartner Inc., (a través de su analista Brian Burke (2014), la define (en traducción libre del inglés por el autor del trabajo) así: 
... una herramienta para formar conductas, desarrollar habilidades y fomentar la innovación. Combinada con otras tecnologías y tendencias, la gamificación puede producir disrupción en la innovación, gestión eficiente de los empleados, educación y formación, desarrollo personal y fidelización ("engagement") de los clientes (Burke, 2014).

Es decir, que con esta actividad se pretende potenciar la motivación, la concentración, el esfuerzo, la fidelización y otros valores positivos comunes a todos los juegos, para que los miembros de una comunidad (trabajadores de una empresa, estudiantes de un colegio/instituto/universidad, habitantes de una ciudad o un barrio) participen en acciones que en general requieren un esfuerzo de la voluntad.

Según el ya mencionado Burke (2014), las organizaciones deben entender bien el enorme potencial de esta herramienta y sus directivos, a su vez, deben utilizar empresas con experiencia y altos conocimientos para desarrollar sus proyectos de gamificación.

En realidad, la gamificación está en la intersección entre el marketing y la educación con la tecnología y la psicología. El concepto del "juego" ha sido profusamente utilizado en la psicología y en el marketing, pero hasta la introducción de la gamificación en el mundo empresarial no se había analizado en profundidad como una disciplina en sí.

Un juego es una actividad de resolución de problemas con una actitud lúdica (Schell, 2008, p. 37). Una importante característica suya es que la participación es voluntaria, y resulta atractiva, emocionante y competitiva.

\section{Sobre la gamificación como elemento vertebrador de la comunicación interna}

La comunicación interna pretende estar en mejor disposición de alcanzar los objetivos empresariales, de forma que resulte estratégica para que influya y resalte los valores intangibles, los que defiende la empresa, su misión, su cultura, su marca y su reputación.

Según Sara Montiel (2017) en su blog Deusto Formación, los objetivos que debe cumplir un plan de comunicación interna deberían basarse en los cinco pilares fundamentales siguientes:

- Informar. La organización se tiene que mostrar transparente e informar sobre la misión, los principios y valores suyos, y específicamente los valores de la estrategia. 
- Democratizar la comunicación. Permitiendo el diálogo y escuchando se facilita el encuentro para obtener mejores soluciones.

- Potenciar la identidad y el sentimiento de pertenencia de los trabajadores para así retener el talento.

- Implicar y motivar. Involucrar a los miembros de la organización para que todos se sientan partícipes del proyecto común.

- Innovar. Buscar e implementar diferentes medios que permitan la comunicación haciendo uso de nuevas estrategias y herramientas más allá de las tradicionales.

El objetivo básico de la gamificación aplicada a la empresa es estimular la actividad dinámica y frecuente entre sus miembros o trabajadores. Se trata de crear experiencias memorables para sugerir emociones positivas e incrementar el sentido de pertenencia.

Sabina Santacroce (2017) afirma:

La gamificación de la comunicación interna para generar compromiso ("engagement") en los colaboradores es una solución cada vez más frecuente. Esto implica incorporar elementos lúdicos en términos de estructura y contenido de la estrategia de comunicación interna tradicional, para atraer, retener y motivar a la fuerza laboral (2017).

De hecho, existen ya estudios y aplicaciones prácticas del uso de la gamificación como estrategia de marketing interno (Ruizalba et al., 2013); incluso, algunos ya afirman que la gamificación interna mejora el rendimiento, la productividad y el compromiso de los empleados (Observatorio de Recursos Humanos, 2021).

Para diseñar un juego que pueda alcanzar buenos resultados, la empresa debe tener claro cuáles son los objetivos que persigue, a qué colectivo dentro de ella se dirige y sus motivaciones, y elegir una recompensa que se adapte a los objetivos seleccionados.

\section{Trabajo de campo realizado}

Para alcanzar la finalidad exploratoria de este estudio se ha llevado a cabo un trabajo de campo que como objetivo principal pretendía obtener información sobre (i) si los directivos consideran que el teletrabajo dificulta las relaciones interpersonales y aleja a los miembros de la empresa del conocimiento de su situación y (ii) si la gamificación puede ser una herramienta útil para evitarlo, mejorar el conocimiento que los empleados tienen de su empresa y facilitar la difusión de la información; en definitiva, mejorar la comunicación interna mediante la gamificación. 
Además de las preguntas dirigidas a los dos objetivos principales, se ha pretendido también en las entrevistas, como objetivo secundario, constatar el conocimiento que los directivos tienen de los objetivos estratégicos de sus empresas y los medios que utilizan para su difusión entre su personal. Parecía oportuno incluir este análisis, por cuanto ello no depende de la utilización (mayor o menor) que se pueda haber hecho del teletrabajo y ya debía estar ocurriendo antes de la pandemia.

Así que se han realizado entrevistas a profundidad en empresas españolas, todas ellas consideradas pymes (es decir, con menos de 250 empleados) y con relevante actividad de teletrabajo (no solo como consecuencia de la pandemia). Se ha intentado, como criterio básico de selección de la muestra, que las personas entrevistadas pertenezcan a empresas con cierto nivel tecnológico en sus herramientas habituales de trabajo y que resulten representativas de los distintos sectores de la actividad económica (Tabla 1). Finalmente, se obtuvieron respuestas de 13 de ellas y de varios de sus cargos directivos, para un total de 32 entrevistas.

Todos los participantes fueron informados del estudio y se obtuvo su consentimiento para participar en el mismo. Las entrevistas son anónimas y ningún participante ha tenido conocimiento de las respuestas de los demás.

\section{Tabla 1. Tipos de empresas participantes}

\begin{tabular}{|c|l|}
\hline Empresas & \multicolumn{1}{|c|}{ Actividad } \\
\hline 1 & Diseño videojuegos/gamificación \\
\hline 2 & Diseño videojuegos/gamificación \\
\hline 3 & $\begin{array}{l}\text { Diseño, fabricación, comercialización muebles y } \\
\text { hostelería }\end{array}$ \\
\hline 4 & Despacho abogados \\
\hline 5 & Diseño y desarrollo de software \\
\hline 6 & Diseño y desarrollo de software \\
\hline 7 & Marketing on line \\
\hline 8 & Formación on line \\
\hline 9 & Ingeniería/alta tecnología \\
\hline 10 & Transformación digital/servicios TIC \\
\hline 11 & Ingeniería/arquitectura \\
\hline 12 & Transformación digital/servicios TIC \\
\hline 13 & Formación on line \\
\hline
\end{tabular}


El cuestionario está compuesto por cuatro bloques de preguntas para verificar el conocimiento u opinión del equipo directivo sobre (i) la relación interpersonal con el teletrabajo, (ii) su conocimiento de los datos estratégicos de la empresa, (iii) los medios de difusión interna de la información (comunicación) que se utilizan y (iv) la gamificación.

Se trata de verificar con el cuestionario si los directivos creen que existe mayor dificultad en la relación entre personas de la empresa y si estas conocen sus datos estratégicos (lo que puede reforzar su compromiso), y en tal caso, si utilizan la gamificación como medio de comunicación interna para solucionarlo.

Todo ello puede sintetizarse en la Tabla 2:

Tabla 2. Agrupación de preguntas de la entrevista por bloques

\begin{tabular}{|l|l|l|}
\hline & \multicolumn{1}{|c|}{ Variable temática } & \multicolumn{1}{c|}{ Valoración de la situación } \\
\hline Bloque 1 & $\begin{array}{l}\text { El teletrabajo y la relación } \\
\text { interpersonal en la empresa }\end{array}$ & Preguntas cerradas (sí/no) \\
\hline Bloque 2 & $\begin{array}{l}\text { Conocimiento de los objetivos } \\
\text { estratégicos de la empresa }\end{array}$ & $\begin{array}{l}\text { Preguntas abiertas (descripción } \\
\text { de objetivos y con un máximo de tres) }\end{array}$ \\
\hline Bloque 3 & $\begin{array}{l}\text { Utilización de los medios de } \\
\text { comunicación para la difusión interna }\end{array}$ & $\begin{array}{l}\text { Preguntas cerradas (opciones } \\
\text { concretas) }\end{array}$ \\
\hline Bloque 4 & Conocimiento y uso de la gamificación & $\begin{array}{l}\text { Preguntas cerradas (sí/no) y una } \\
\text { abierta (sin límite de extensión) }\end{array}$ \\
\hline
\end{tabular}

Las respuestas "sí/no" no bloquean las preguntas siguientes, a excepción de un "no" en relación con el conocimiento de la gamificación, en cuyo caso el entrevistado no puede indicar su utilidad ni en qué sentido la utilizaría.

Las entrevistas fueron realizadas (menos 2) por medios on line entre los días 2 y 13 de noviembre de 2020. Todas ellas se han considerado completas y válidas y han permitido obtener los resultados que se describen en el punto siguiente.

\section{Análisis de los resultados}

En el bloque 1, 19 directivos (59,4\%) creen que el teletrabajo dificulta las relaciones interpersonales de la empresa y 16 de ellos (el 50 \% exactamente) creen que, además, aleja a las personas del conocimiento de la situación de la compañía y de sus objetivos. Interesante la lectura inversa de esta cuestión: es decir, que el 50 \% de los equipos directivos no consideran 
que el teletrabajo deteriore el conocimiento de los colaboradores sobre la empresa (Tabla 3):

Tabla 3. Respuestas a preguntas del bloque 1

\begin{tabular}{|c|l|c|c|c|c|}
\hline \multirow{5}{*}{} & \multicolumn{1}{c|}{ Pregunta } & \multicolumn{2}{|c|}{ Si } & \multicolumn{2}{c|}{ No } \\
\cline { 2 - 6 } Bloque 1 & $\begin{array}{l}\text { ¿El teletrabajo dificulta } \\
\text { la relación interpersonal } \\
\text { en la empresa? }\end{array}$ & 19 & $59,4 \%$ & 13 & $40,6 \%$ \\
\cline { 2 - 6 } & $\begin{array}{l}\text { ¿El teletrabajo aleja a las } \\
\text { personas del conocimiento } \\
\text { de la empresa? }\end{array}$ & 16 & $50,0 \%$ & 16 & $50,0 \%$ \\
\hline
\end{tabular}

En el bloque 2 (preguntas 3, 4 y 5, conocimiento sobre la propia empresa), figuraban dos preguntas abiertas y una cerrada. Este bloque trataba de obtener el grado de conocimiento que el equipo directivo tenía sobre los objetivos estratégicos de su propia empresa (como ejemplo de que la difusión de datos estratégicos ya se está haciendo) y, en el caso de varias entrevistas a sus directivos, el grado de coincidencia entre ellos para dicha información. Este aspecto, como ya se ha mencionado, no tiene por qué estar vinculado al teletrabajo (por causa de la pandemia o por decisión de la empresa) y pretendía saber si aquel conocimiento ya existía y cuál era el mecanismo de difusión utilizado.

La primera pregunta del bloque 2 solicitaba al entrevistado que definiera la misión de su empresa y que indicara si esta correspondía a la mencionada en la página web. Todos los entrevistados (32) describieron la misión de su empresa, pero solo seis (18,7 \%) conocían la descripción publicada en su propia web. 16 entrevistados (50 \%) afirmaron no conocer o recordar lo publicado en su web ("creo recordar sin mirar que no la tenemos inscrita en la página web", "no", "no exactamente") y nueve afirmaron que lo saben aproximadamente ("palabra arriba o abajo"). Hay cinco empresas cuya misión no está publicada en la web o en otros documentos informativos de carácter público, sin que se pueda concluir que ello se deba a una voluntad deliberada (quizá falta de actualización de la web o simple descuido).

Tampoco se ha detectado un patrón en la descripción de la misión. Es decir, cada persona y cada empresa utiliza su propio modelo de expresión, a veces largo y con multitud de conceptos que se entrelazan en una misma frase, y otros más conceptuales, con los mensajes que quieren transmitir separados por líneas. Esto ocurre incluso entre los miembros de una misma empresa, 
que no siempre coinciden en sus definiciones o conceptos básicos de la misión y, por tanto, tampoco en la forma de expresarla.

En este mismo bloque 2, la segunda pregunta pretendía ver si los entrevistados conocían los tres objetivos fundamentales que la empresa tenía para el ejercicio en curso, con lo que se obtendría otro dato (además del anterior sobre la misión) del grado de conocimiento estratégico de la compañía. 27 de ellos $(84,4 \%$ ) describieron tres objetivos de la empresa para un ejercicio completo (en curso o el siguiente) y cinco (15,6\%) solo dos, si bien en el caso de varias entrevistas en una misma empresa no hubo coincidencia entre los indicados por el máximo responsable (CEO o director general) y sus más directos colaboradores (director técnico, comercial o de marketing).

Esta falta de coincidencia podría, no obstante, achacarse a una interpretación de la pregunta, en el sentido de que el entrevistado indicó los objetivos de su departamento o función y no los de la empresa; a pesar de esta consideración, al menos parcialmente deberían coincidir o ser coherentes entre sí.

No se trataba de analizar la calidad de las respuestas en el sentido técnico, sino de verificar que existían objetivos definidos y compartidos por todos los miembros del equipo directivo. De la forma y expresión de las respuestas podría desprenderse que los objetivos no están claramente definidos ni se difunden regularmente para que todos los tengan presentes permanentemente y puedan ser repetidos automáticamente por cualquier miembro del equipo directivo. Antes bien, cada uno lo hace suyo y lo expresa a su manera o, simplemente, lo interpreta y lo prioriza según sus funciones.

Así las cosas, de este bloque 2 se desprende una falta de unidad generalizada entre los miembros de los equipos directivos en torno a la misión de su propia compañía y a los objetivos estratégicos del ejercicio.

Finalmente, y todavía dentro de este bloque, prácticamente un tercio de los directivos (11 de ellos) no sabía si el personal a su cargo era conocedor de los objetivos de la empresa, lo que denota que, al menos en estos casos, no ha habido difusión interna de aquellos o hay pobreza en los procesos de comunicación.

El bloque 3 del cuestionario pretendía averiguar cuáles son los medios de comunicación interna de estas empresas para la difusión de su información y los temas básicos que tratan.

Para la comunicación interna o difusión de la información (bloque 3, preguntas 6 y 7) todos utilizan (32 casos) las reuniones específicas, que complementan con notas informativas específicas (cinco casos), publicación 
frecuente de newsletter (cuatro casos) y redes sociales (cuatro casos). Como actividades colectivas, siete participantes (21,8 \%) llevan a cabo ejercicios de team building y solo dos empresas han manifestado que mantienen eventos anuales con toda o parte importante de la plantilla.

Se ha sintetizado el resultado en la Tabla 4:

Tabla 4. Respuestas a pregunta del bloque 3: medios para difusión de la información

\begin{tabular}{|c|c|c|c|c|c|c|c|c|c|c|c|}
\hline \multicolumn{2}{|c|}{$\begin{array}{c}\text { Reuniones } \\
\text { especificas }\end{array}$} & $\begin{array}{c}\text { Notas } \\
\text { informativas } \\
\text { específicas }\end{array}$ & \multicolumn{2}{|c|}{ Newsletter } & \multicolumn{2}{|c|}{$\begin{array}{c}\text { Team } \\
\text { building }\end{array}$} & \multicolumn{2}{|c|}{$\begin{array}{c}\text { Redes } \\
\text { Sociales }\end{array}$} & \multicolumn{2}{|c|}{ Evento anual } \\
\hline 32 & $100 \%$ & 5 & $16 \%$ & 4 & $13 \%$ & 7 & $22 \%$ & 4 & $13 \%$ & 2 & $6 \%$ \\
\hline
\end{tabular}

De las reuniones específicas para comunicar la situación de la empresa y sus objetivos estratégicos, sólo 3 de ellas utilizan la videollamada o videoconferencia.

No parece, pues que ninguna de las empresas entrevistadas utilice las opciones que hoy en día ofrecen las tecnologías para facilitar la comunicación, ni siquiera las propias redes sociales, sólo usada por 4 de ellas.

Todavía en el Bloque 3, se incluía la pregunta sobre el tipo de información que se trasmitía habitualmente por los medios anteriores. Se trataba también de una pregunta cerrada con cuatro opciones; de las empresas entrevistadas, el 81,2 \% de ellas concentran su información en la transmisión de valores y principios (26 casos), el 65,6 \% en objetivos estratégicos (21 casos) y el 37,5\% en criterios ejecutivos ( 12 casos). Solamente en 2 casos se han transmitido otro tipo de informaciones o datos, lo que significa que la práctica totalidad de las empresas concentran toda la información a transmitir en los tres conceptos indicados.

De forma esquemática se pueden sintetizar las respuestas en la Tabla 5:

Tabla 5. Respuestas a pregunta del bloque 3: temática de la comunicación

\begin{tabular}{|c|c|c|c|c|c|c|c|}
\hline \multicolumn{2}{|c|}{$\begin{array}{c}\text { Objetivos } \\
\text { estratégicos }\end{array}$} & \multicolumn{2}{|c|}{ Valores y principios } & \multicolumn{2}{c|}{ Criterios ejecutivos } & \multicolumn{2}{c|}{ Otros } \\
\hline 21 & $65,6 \%$ & 26 & $81,3 \%$ & 12 & $37,5 \%$ & 2 & $6,3 \%$ \\
\hline
\end{tabular}


En ambas preguntas del bloque 3 (medios utilizados y temática de la comunicación), cada persona podía utilizar varias respuestas, es decir, indicar varios medios de comunicación, e incluir varias temáticas en ellos. Dicho de otra forma: el sentido negativo de la respuesta significaría que 11 personas no comunican (de ninguna manera) sus objetivos estratégicos, seis de ellas no lo hacen con los principios y valores, y 20 no transmiten sus criterios ejecutivos.

Finalmente, el bloque 4 pretendía analizar el grado de conocimiento que sobre la gamificación tenía cada entrevistado y, en caso positivo, la experiencia vivida con ello y el potencial uso que podrían darle en su empresa.

En cuanto al conocimiento y uso o potencial uso de la gamificación en la empresa, el $87,5 \%$ de los entrevistados (28) dijeron conocerla, pero solo seis de ellos (es decir, un 18,75\%) la ha utilizado en su empresa actual o en una anterior (Tabla 6). La pregunta no permite discernir si el entrevistado entendió que esta gamificación se refería a usos empresariales y no a otros exclusivamente lúdicos o propios del ocio. Finalmente, cuatro de ellos ni siquiera saben en qué consiste la gamificación, por lo que no han podido aportar nada en relación con su potencial uso en sus empresas.

De forma casi unánime, los entrevistados coinciden en que la gamificación puede ser una herramienta muy útil tanto para la comunicación interna como para la cohesión de los equipos (28 y 26 casos, respectivamente), pese al poco uso que habían hecho de ella hasta el momento de la entrevista (Tabla 6).

Tabla 6. Respuestas a preguntas del bloque 4

\begin{tabular}{|c|c|c|c|c|c|c|c|}
\hline \multicolumn{2}{|c|}{$\begin{array}{l}\text { Conocen la } \\
\text { gamificación }\end{array}$} & \multicolumn{2}{|c|}{$\begin{array}{l}\text { La han utilizado } \\
\text { alguna vez }\end{array}$} & \multicolumn{2}{|c|}{$\begin{array}{l}\text { Es útil para la } \\
\text { comunicación } \\
\text { interna }\end{array}$} & \multicolumn{2}{|c|}{$\begin{array}{c}\text { Puede mejorar } \\
\text { la cohesión de la } \\
\text { empresa }\end{array}$} \\
\hline 28 & $87,5 \%$ & 6 & $18,8 \%$ & 28 & $87,5 \%$ & 26 & $81,3 \%$ \\
\hline
\end{tabular}

La última pregunta de este bloque era abierta y permitía con libertad que cada entrevistado ofreciera su propia opinión, a la vista de la utilidad de la gamificación como herramienta de comunicación evidenciada en sus respuestas anteriores (Tabla 6), sobre cómo utilizarla y a qué objetivos la dirigiría.

Como ya se dijo, se validaron 28 respuestas (el 87,5 \%), por cuanto las cuatro que desconocen la gamificación no podían contestar esta última pregunta. 
Se han clasificado las respuestas en función de los objetivos que cada entrevistado pretendía cumplir con el uso de la gamificación. Se destaca que 14 (el 43,7 \%) dicen no saber cómo enfocarla ("desconozco cómo enfocarlo", "no sé", "no conozco demasiado del tema", "preferiría alguna actividad presencial, pero la situación actual no lo facilita", "me falta conocimiento más profundo", "habría que pensarlo detenidamente"), y tres de ellos lo solucionarían poniéndose en manos de expertos en la materia.

El 34,4\%, es decir, 11 de los entrevistados, utilizaría la gamificación para el desarrollo profesional ("la utilizamos en el training técnico, pero hasta ahora no en la transmisión de información estratégica", "para el desarrollo de su carrera profesional en la compañía", "asociada a las palancas de liderazgo"); siete de ellos (21,9 \%) para mayor conocimiento de la empresa y sus productos ("conocer mejor las características de los productos que fabricamos y vendemos", "facilitar procesos de aprendizaje"), por cuanto se inclinan por el uso más personal y lúdico buscando la relación y la cohesión ("proponiendo desafíos personales", "que permitan el crecimiento personal y equipo-empresa desarrollando objetivos individuales").

\section{Hallazgos}

Entre los resultados obtenidos cabe destacar los siguientes hallazgos:

- Solo la mitad de los entrevistados admite que el teletrabajo está disminuyendo el conocimiento que los empleados tienen de la empresa y casi el 60 \% cree que dificulta la interrelación de las personas. Esta información resultaba de interés para que en caso de que así fuera, se pudiera conocer qué mecanismos de solución estaban implantando las empresas y en qué medida la gamificación estaba jugando un papel significativo. Con el resultado observado, no parece que, al menos por ahora, sea un factor de preocupación general en los equipos directivos.

- No existe en estas empresas una estrategia adecuada de comunicación interna, por cuanto entre los mismos miembros de los equipos directivos no se ha encontrado coincidencia clara de los objetivos y valores estratégicos. No puede desprenderse de este estudio que ello se deba al alejamiento por el teletrabajo o si ya existía desde antes.

- Pese a ser empresas que utilizan profusamente para el desarrollo de sus actividades las tecnologías digitales e informáticas, ninguna de ellas ha puesto en práctica procesos de gamificación en sus estrategias de comunicación interna. Las empresas analizadas siguen utilizando métodos tradicionales, como las reuniones para comunicación de resultados y objetivos y las newsletter como elemento de la comunicación escrita. Con ellos tratan de difundir los objetivos y valores estratégicos, 
así como los criterios ejecutivos, aunque con un éxito relativo, como se ha visto. El no uso de la gamificación para la comunicación interna ha impedido profundizar en las formas de utilización y sus objetivos.

\section{Conclusiones}

El objetivo principal de este estudio consistía en averiguar si el alejamiento físico de los miembros de una estructura organizativa (pyme) de sectores tecnológicos o digitales de entorno VUCA debido al teletrabajo dificultaba las relaciones interpersonales y el conocimiento de los objetivos y valores estratégicos de la empresa, y si esto llevaría a la disminución del sentido de pertenencia de los trabajadores; asimismo, en qué medida se estaba utilizando la gamificación como elemento clave en los procesos de comunicación interna para paliarlo o solucionarlo.

Los hallazgos del estudio muestran que la mitad de los directivos no considera que el teletrabajo genere alejamiento, por lo que no han tomado ninguna acción correctora, pese a que existe poca coincidencia en el conocimiento de sus miembros sobre los datos clave de cada compañía. La otra mitad piensa lo contrario, pero en general tampoco han iniciado procesos correctores. No parece, pues, que este problema resulte de gran preocupación para los directivos de este tipo de empresas.

Aunque este estudio pretendía analizar también el grado de implantación de la gamificación en este tipo de empresas y cómo la estaban utilizando, el hecho de que ninguna de ellas la haya puesto en práctica ha limitado enormemente los hallazgos y conclusiones. Esto resulta indicativo de la poca penetración que tiene todavía la gamificación en las pymes y, quizá, lo prematuro de este trabajo.

Bien es cierto que todos los entrevistados que conocían la gamificación $(87,5 \%)$ se mostraron de acuerdo con que puede ser una buena herramienta para la comunicación interna y para mejorar el conocimiento de la empresa y la cohesión de sus trabajadores, aunque necesitarán soporte de profesionales externos.

La limitada extensión de la muestra y el carácter exploratorio del trabajo, si bien aporta elementos indiciarios de la problemática, impiden establecer conclusiones de ámbito general, por lo que debería complementarse en un futuro próximo con una investigación correlacional de una muestra mucho más amplia y una metodología más dirigida a la gamificación, de manera que se pueda establecer una cuantificación de su uso y eficacia en la comunicación interna de las pymes, con el fin de mejorar las interrelaciones 


\section{Referencias}

Aquino, Y. (2019). Serious Games: las empresas y la gamificación de las cosas. Universidad de Murcia.

Burke, B. (2014). Gamify: how gamification motivates people to do extraordinary things. Routledge.

Chang, C. (2015). Computers in human behaviour. Elsevier.

Coombs, T. (2019). Transmedia storytelling: a potentially vital resource for CSR communication. Corporate Communications: An International Journal, 24(2), 351-367. https:// doi. org/10.1108/CCIJ-11-2017-0114

Courtney, H. Kirkland, J. Viguerie, P., (2000). Strategy under uncertainty. McKinsey. The Changing Landscape (81-90). https://www. mckinsey.com/ /media/McKinsey/Featured\%20Insights/Managing\%20In\%20Uncertainty/Strategy\%20under\%20uncertainty/ Strategy\%20under\%20uncertainty.pdf?shouldIndex=false

Cuenca, J. y Verazzi, L. (2018). Guía fundamental de la comunicación interna. Editorial UOC. Curcio, C. (2006). Metodología de la investigación. McGraw Hill.

Dudalia. (2017). Tipos de redes sociales [Respuesta]. https://www.tiposde.org/internet/87-tipos-de-redes-sociales/\#ixzz6kxw26T3a

Gallego Durán, F., Villagrá-Arnedo, C., Satorre, R., Compañ, P., Molina, R. y Llorens, F. (2014). Panorámica: serious games, gamification y mucho más. https: / dialnet.unirioja.es/ servlet/articulo? codigo $=5828053 \&$ orden $=0$ \&info=link

Gil, G. y Martínez Arias, M. (2001). Metodología de encuestas. En M. J. Navas (Coord.), Métodos, diseños y técnicas de investigación sociológica (pp. 379-436). Universidad Nacional de Educación a Distancia [UNED].

Goodpaster, K. E. (2006). Conscience and corporate culture. Wiley-Blackwell.

Hunicke, R., LeBlanc, M. y Zubeck, R. (s. f.). MDA: a Formal Approach to Game Design and Game Research [PDF]. http://www.cs.northwestern.edu/ hunicke/MDA.pdf

INE (Instituto Nacional de Estadística), Boletín Informativo, 02/2020. https://www.ine.es/ SS/Satellite? L=es_ES\&C=INECifrasINE_C\&cid=1259952649680\&p=1254735116567\&pagename=ProductosYServicios\%2FINECifrasINE_C\%2FPYSDetalleCifrasINE

Kail, E. (2011, enero 6). Leading effectively in a VUCA environment: a is for ambiguity. Harvard Business Review. “http://blogs.hbr.org/frontline-leadership/2011/01/leading-effectively-in-a-vuca-1.html"

Kingsinger, P. y Walch, K. (2012). Living and leading in a VUCA world.

Lawrence, K. (2013). Developing Leaders in a VUCA Environment, UNC Kenan-Flagler Business School.

Lévy, P. (2004), Inteligencia colectiva: por una antropología del espacio. Organización Panamericana de la Salud. 
Marczewski, A. (2015). User types. En A. Marczewski, Even Ninja Monkeys like to play: gamification, game thinking and motivational design. Createspace Independent Publishing Platform.

Marina, J. A. (2016). Objetivo: generar talento. Editorial Conecta.

McGonigal, J. (2011). Reality is broken: why games make us better and how they can change the world. Penguin Books.

Méndez, A. I. (2007). Metodologías y técnicas de investigación aplicadas a la comunicación [PDF]. https://metodouces.files.wordpress.com/2015/09/comunicacion_mendez.pdf

Montiel, S. (2017, julio 26). Objetivos y beneficios de la comunicación interna. Deusto Formación [Blog]. https://www.deustoformacion.com/blog/gestion-empresas/objetivos-beneficios-comunicacion-interna

Observatorio de Recursos Humanos. (2021, junio 5). La gamificación interna mejora el rendimiento, la productividad y el engagement de los empleados. "https: / / www.observatoriorh.com/comunicacion-y-branding/gamificacion-interna-rendimiento-productividad-engagement-empleados.html"

Pineda-Martínez, P. y Ruiz-Mora, I., (2019). Comunicación interna y narrativas transmedia nuevas estrategias para la empresa responsable. Estudio de caso de Telefónica. El Profesional de la Información, 28(5).

Randstad (2021). La evolución del teletrabajo en España. “https://www.randstad.es/tendencias360/la-evolucion-del-teletrabajo-en-espana/"

Rosser, C. (2003). Jefes, clientes, colaboradores, pareja. FC Editorial (España)

Ruizalba, J.L., Navarro, F., y Jiménez, S. (2013). Gamificación como estrategia de marketing interno. Universidad de Málaga.

Sánchez Burón, A. y De la Morena Taboada, M. (2002). Pensamiento creativo. Enciclopedia de la Pedagogía, 1.

Santacroce, S. (2017). 5 razones para potenciar la comunicación interna a través de la “Gamificación”. PuroMarketing. https: / / www.puromarketing.com/30/28697/razones-para-potenciar-comunicacion-interna-traves-gamificacion.html

Schell, J. (2008). The art of game design: a book of lenses. Morgan Kaufmann Publishers.

Stiglitz, J. y Greenwald, B. (2016). La creación de una sociedad del aprendizaje. La Esfera de los Libros.

Viñarás Abad, M. (2020). La comunicación interpersonal y la comunicación interna en las empresas: un análisis desde la profesión y la universidad. Comunicación y Hombre, (16), 335-354. https:// portalderevistas.ufv.es/index.php/comunicacionyhombre/article/ view/607/603Informativo, 02/2020.https://www.ine.es/ss/Satellite? L=es_ES\&C=INECifraSINE_C\&cid=1259952649680\&p=1254735116567\&pagename=ProductosYServicios\%2FINECifrasINE_C\%2FPYSDetalleCifrasINE

Kail, E. (2011, enero 6). Leading effectively in a VUCA environment: a is for ambiguity. Harvard Business Review. http:// blogs.hbr.org/frontline-leadership/2011/01/leading-effectively-in-a-vuca-1.html

Kingsinger, P. y Walch, K. (2012). Living and leading in a VUCA world.

Lawrence, K. (2013). Developing Leaders in a VUCA Environment, UNC Kenan-Flagler Business School. 
Lévy, P. (2004), Inteligencia colectiva: por una antropología del espacio. Organización Panamericana de la Salud.

Marczewski, A. (2015). User types. En A. Marczewski, Even Ninja Monkeys like to play: gamification, game thinking and motivational design. CreateSpace Independent Publishing Platform.

Marina, J. A. (2016). Objetivo: generar talento. Editorial Conecta.

McGonigal, J. (2011). Reality is broken: why games make us better and how they can change the world. Penguin Books.

Méndez, A. I. (2007). Metodologías y técnicas de investigación aplicadas a la comunicación [PDF]. https://metodouces.files.wordpress.com/2015/09/comunicacion_mendez.pdf

Montiel, S. (2017, julio 26). Objetivos y beneficios de la comunicación interna. Deusto Formación [Blog]. https://www.deustoformacion.com/blog/gestion-empresas/objetivos-beneficios-comunicacion-interna

Observatorio de Recursos Humanos. (2021, junio 5). La gamificación interna mejora el rendimiento, la productividad y el engagement de los empleados. https://www.observatoriorh.com/comunicacion-y-branding/gamificacion-interna-rendimiento-productividad-engagement-empleados.html

Pineda-Martínez, P. y Ruiz-Mora, I., (2019). Comunicación interna y narrativas transmedia nuevas estrategias para la empresa responsable. Estudio de caso de Telefónica. El Profesional de la Información, 28(5).

Randstad (2021). La evolución del teletrabajo en España. https://www.randstad.es/tendencias360/ la-evolucion-del-teletrabajo-en-espana/

Rosser, C. (2003). Jefes, clientes, colaboradores, pareja. FC Editorial (España)

Ruizalba, J.L., Navarro, F., y Jiménez, S. (2013). Gamificación como estrategia de marketing interno. Universidad de Málaga.

Sánchez Burón, A. y De la Morena Taboada, M. (2002). Pensamiento creativo. Enciclopedia de la Pedagogía, 1.

Santacroce, S. (2017). 5 razones para potenciar la comunicación interna a través de la "Gamificación". PuroMarketing. https://www.puromarketing.com/30/28697/razones-para-potenciar-comunicacion-interna-traves-gamificacion.html

Schell, J. (2008). The art of game design: a book of lenses. Morgan Kaufmann Publishers.

Stiglitz, J. y Greenwald, B. (2016). La creación de una sociedad del aprendizaje. La Esfera de los Libros.

Viñarás Abad, M. (2020). La comunicación interpersonal y la comunicación interna en las empresas: un análisis desde la profesión y la universidad. Comunicación y Hombre, (16), 335-354. https:// portalderevistas.ufv.es/index.php/comunicacionyhombre/article/ view/607/603 